\title{
On Application of Matrix-Vector Operations for Solving Constrained Optimization Problems
}

\author{
${ }^{1}$ T. E. Olaosebikan, ${ }^{2}$ S. A. Olorunsola and ${ }^{3}$ K. J. Adebayo \\ ${ }^{1,2,3}$ Department of Mathematical Sciences, Ekiti State University, Ado Ekiti, Nigeria.
}

\begin{abstract}
This paper discusses the application of matrix-vector operations technique for solving constrained optimization problems. The method is aimed at circumventing the computational rigours undergone using the simplex and revised simplex method in solving this class of problems with aim of reducing the computer memory space occupied by the methods. In other to achieve this, a straightforward and simple to handle matrix-vector operations algorithm has been developed to solve the same constrained problems. Numerical results show some improvements compared with the classical method.
\end{abstract}

Keywords: Constrained Optimization Problem, Vector Operations and Matrix Operations.

\section{Introduction}

The general optimization problem to be considered is of the form by [1] and [2] as:

$\begin{array}{clr}\text { Optimize: } & f(x) & \text { (objective function) } \\ \text { Subject to: } & & 1.1 \\ & h_{i}(x)=0 i=1,2, \ldots, m_{1} & \text { (equality constraint) } \\ g_{j}(x) \geq 0 j=1,2, \ldots, m_{2} & \text { (inequality constraint) } & 1.2 \\ x \geq 0 & \text { (non-negative condition) } & 1.3 \\ & & \end{array}$

where $x$ is a vector of $\mathrm{n}$ variables $\left(x_{1}, x_{2}, \ldots, x_{n}\right), h_{i}(x)$ is a vector of equations of dimension $m_{1}$, and $g_{j}(x)$ is a vector of inequality of dimension $m_{2}$, and the total number of constraints $m=\left(m_{1}+m_{2}\right)$. Methods for solving this model have been developed, tested and successfully applied to many important problems of scientific and economic interest. However, in spite of the proliferation of the methods, there is no universal method for solving all optimization problems which calls for application of matrix-vector operations techniques to solving (1.1) through (1.4).

\section{Computational Procedure of Matrix-Vector Operations}

Before discussing the computational procedure of matrix-vector operations to solving constrained optimization problems which is our major concern, it is expedient to show the following theorems which will be advantageous in other to get the clear picture of what the analysis is all about.

\section{Theorem 1}

(a) Suppose $M$ is an $n x n$ non-singular matrix then:

$$
M(\operatorname{adj}(M))=(\operatorname{adj}(M)) M=\operatorname{det}(M) l_{n}
$$

(b) If $M$ is $n x n$ non-singular matrix with $\operatorname{det}(M) \neq 0$ then:

$$
M^{-1}=\frac{1}{d e t}(M(\operatorname{adj}(M))
$$

where $l_{n}$ is an identity matrix.

Proof:

(a) To show (2.1),

Let $M=\left[\begin{array}{ccc}a_{11} & \cdots & a_{1 n} \\ \vdots & \ddots & \vdots \\ a_{n 1} & \cdots & a_{n n}\end{array}\right]$

and

let $W=\left[W_{i j}\right]$

be the cofactor matrix then:

$\operatorname{adj}(M)=\left[\begin{array}{ccc}w_{11} & \cdots & w_{n 1} \\ \vdots & \ddots & \vdots \\ w_{1 n} & \cdots & w_{n n}\end{array}\right]$.

Considering the product of (2.3) and (2.5), we observe that the entry in the $i t h$ row and $j$ th column is:

$a_{i 1} w_{i 1}+a_{i 2} w_{i 2}+\ldots+a_{i n} w_{i n}$ 
Now if $i=j$, then this expression is the cofactor expansion of $\operatorname{det}(M)$ along the $i t h$ of $M$. But if $i \neq j$, the factors of this product come from different rows of $M$ and so the expression has a value of zero. This means that:

$$
\begin{aligned}
M(\operatorname{adj}(M) & =\left[\begin{array}{ccc}
\operatorname{det}(M) & \cdots & 0 \\
\vdots & \ddots & \vdots \\
0 & \cdots & \operatorname{det}(M)
\end{array}\right] \\
& =\operatorname{det}(M) l_{n}
\end{aligned}
$$

Similarly, the entry in the ith row and jth colum
$W_{1 i} a_{1 i}+W_{2 i} a_{2 i}+\ldots+W_{n i} a_{n i}=\operatorname{det}(M)$

for $i=j$. For $i \neq j$, hence $(2.1)$ holds.

(b) From (2.1) we have:

$(\operatorname{adj}(M)) M=\operatorname{det}(M) l_{n}$.

Since $M$ is non-singular and therefore $\operatorname{det}(M) \neq 0$, we have

$\frac{1}{\operatorname{det}(M)}=\frac{1}{\operatorname{det}(M) l_{n}}$

Or

$\frac{1}{\operatorname{det}(M)}[M(\operatorname{adj}(M))]=l_{n}$

$M\left[\frac{1}{\operatorname{det}(M)}\right] \operatorname{adj}(M)=l_{n}$

Multiplying (2.12) through by $M^{-1}$ and (2.2) holds

Theorem 2

Suppose $B$ is the transition matrix from a basis $\left(e_{1}\right)$ to a basis $\left(f_{1}\right)$ for a vector space $V$. Then, for any vector $w \in V$,

$\mathrm{B}[W]_{f}=[W]_{e}$

Hence:

$[W]_{f}=B^{-1}[W]_{e}$

Proof:

The proof is presented by illustration. Consider the case of a 3-dimentional vector space. Let $B$ be the transition matrix from basis $\left(e_{1}, e_{2}, e_{3}\right)$ of $V$ to another basis $\left(f_{1}, f_{2}, f_{3}\right)$ of $V$. Then:

$f_{1}=a_{1} e_{1}+a_{2} e_{2}+a_{3} e_{3}$

$\left.f_{2}=b_{1} e_{1}+b_{2} e_{2}+b_{3} e_{3}\right\}$

$\left.f_{3}=c_{1} e_{1}+c_{2} e_{2}+c_{3} e_{3}\right\}$

$B=\left[\begin{array}{lll}a_{1} & a_{2} & a_{3} \\ b_{1} & b_{2} & b_{3} \\ c_{1} & c_{2} & c_{3}\end{array}\right]$,

hence, the transition matrix of (2.16) is:

$B=\left[\begin{array}{lll}a_{1} & b_{1} & c_{1} \\ a_{2} & b_{2} & c_{2} \\ a_{3} & b_{3} & c_{3}\end{array}\right]$.

Now, let $w \in V$ such that:

$W=k_{1} f_{1}+k_{2} f_{2}+k_{1} f_{3}$

substituting (2.15)into (2.18), we have:

$[W]_{e}=k_{1}\left(a_{1} e_{1}+a_{2} e_{2}+a_{3} e_{3}\right)+k_{2}\left(b_{1} e_{1}+b_{2} e_{2}+b_{3} e_{3}\right)+k_{3}\left(c_{1} e_{1}+c_{2} e_{2}+c_{3} e_{3}\right)$

Thus:$$
=\left(a_{1} k_{1}+b_{1} k_{2}+c_{1} k_{3}\right) e_{1}+\left(a_{2} k_{1}+b_{2} k_{2}+c_{2} k_{3}\right) e_{2}+\left(a_{3} k_{1}+b_{3} k_{2}+c_{3} k_{3}\right) e_{3} \text {. }
$$

$[W]_{f}=\left[\begin{array}{l}k_{1} \\ k_{2} \\ k_{3}\end{array}\right]$

and

$[W]_{e}=\left[\begin{array}{lll}a_{1} k_{1} & b_{1} k_{2} & c_{1} k_{3} \\ a_{2} k_{1} & b_{2} k_{2} & c_{2} k_{3} \\ a_{3} k_{1} & b_{3} k_{2} & c_{3} k_{3}\end{array}\right]$.

Now, multiplying (2.17) and (2.20) gives:

$B[W]_{f}=\left[\begin{array}{lll}a_{1} k_{1} & b_{1} k_{2} & c_{1} k_{3} \\ a_{2} k_{1} & b_{2} k_{2} & c_{2} k_{3} \\ a_{3} k_{1} & b_{3} k_{2} & c_{3} k_{3}\end{array}\right]$ 
$B[W]_{f}=[W]_{e}$.

Multiplying $(2.23)$ by $B^{-1}$, we have:

$[W]_{f}=B^{-1}[W]_{e}$.

(2.24) satisfies the theorem.

With the theorems above, the following derivations will be useful. Also, from our knowledge of simplex and revised simplex methods, this will lead us to the next concept to be discussed in what follows:

Let the column vectors $P_{1}, P_{2}, \ldots, P_{n}$ denote the original columns of $X_{1}, X_{2}, \ldots, X_{n}$ and let the column vector $b$ represent the right-hand side constants.

Determine the basic variable and define a basis matrix B whose elements are the original columns of the basic variables.

Obtain the inverse of the basis matrix $B, B^{-1}$.

To determine the non basic variable that enters the basis and the basic variable that leaves the basis, the following steps are carried-out:

$$
\begin{aligned}
\left(\overline{C_{J}}-\overline{Z_{J}}\right) & =C_{j}-\sum C_{B} a_{i j} \\
& =C_{j}-C_{B} \sum a_{i j} \\
& =C_{j}-C_{B} \bar{P}_{J}
\end{aligned}
$$

but $\quad \bar{P}_{J}=B^{-1} P_{j}$ (pivot column)

Putting (2.27) in (2.26), we obtain:

$\left(\overline{C_{J}}-\overline{Z_{J}}\right)=C_{j}-C_{B} B^{-1} P_{j}$

Let the vector multiplier be:

$\pi=C_{B} B^{-1}$

Putting (2.29) in (2.28), we obtain:

$\overline{C_{J}}-\overline{Z_{J}}=C_{j}-\pi P_{j}$ for all $j$

To get the constant:

$$
\bar{b}=B^{-1} b
$$

We calculate the pivot of any entering variable by (2.27) and to get the outgoing variable,

Ratio, $\mathrm{R}$, we use

$$
R=\frac{b}{\overline{P_{J}}}
$$

Step 1: Transform the problem into standard optimization form

\section{The Matrix-Vector Operations Algorithm}

Step 2: Get the column vectors from the problem $\left(P_{1}, P_{2}, \ldots, P_{n}\right)$

Step 3: Determine the initial basis $B$

Step 4: Calculate the inverse of the matrix $B$

Step 5: Determine the entering variable using (2.29) and (2.30) respectively.

Step 6: Determine the pivot column with (2.27)

Step 7: Determine the constant with (2.31)

Step 8: Determine the leaving variable by calculating the ratio using (2.32)

Step 9: Go back to step3 and continue until step 5 becomes all negative or positive for maximization or otherwise for minimization. Then step 7.

Remark: (1). For maximization problem, pick the most positive value of (2.30) and the least positive value of (2.32) until all the values of (2.30) becomes negative. Then, compute (2.31) for optimal solution. (2). For minimization problem, pick the least positive in case all the values are positive or most negative value in case all the values are negative from (2.30) and the most positive or least positive value of (2.32) until all the values of (2.30) yields all positive. Then, compute (2.31) for optimal solution.

\section{Computational Results}

The following problems were evaluated using the afore algorithm thus:

(P1) Maximize: $Z=4 x_{1}+3 x_{2}+6 x_{3}$

Subject to: $\quad 2 x_{1}+3 x_{2}+2 x_{3} \leq 440$

$4 x_{1} \quad+3 x_{3} \leq 470$

$2 x_{1}+5 x_{2} \leq 430$

$x_{1}, x_{2}, x_{3} \geq 0$

(P2) Maximize: $Z=5 x_{1}+x_{2}+3 x_{3}+2 x_{4}$

Subject to: $\quad 2 x_{1}+3 x_{2}+6 x_{3}+x_{4} \geq 6$

$6 x_{1}+3 x_{2}+5 x_{3}+3 x_{4} \geq 8$

$3 x_{1}+6 x_{2}+x_{3}+3 x_{4} \geq 4$

$x_{1}, x_{2}, x_{3}, x_{4} \geq 0$ 
(P3) Minimize: $Z=6 x_{1}+3 x_{2}+4 x_{3}$

Subject to: $\quad x_{1}+6 x_{2}+x_{3}=10$

$$
2 x_{1}+3 x_{2}+x_{3}=15
$$

$$
x_{1}, x_{2}, x_{3} \geq 0
$$

(P4) Maximize: $Z=3 x_{1}+4 x_{2}+x_{3}+5 x_{4}$

Subject to: $\quad 8 x_{1}+3 x_{2}+2 x_{3}+4 x_{4} \leq 10$

$$
\begin{array}{r}
2 x_{1}+5 x_{2}+x_{3}+4 x_{4} \leq 5 \\
x_{1}+2 x_{2}+5 x_{3}+x_{4} \leq 6 \\
x_{1}, x_{2}, x_{3}, x_{4} \geq 0
\end{array}
$$

(P5) Maximize: $Z=2 x_{1}+4 x_{2}+x_{3}$

Subject to: $\quad x_{1}+x_{2}+x_{3} \geq 8$

$$
\begin{aligned}
x_{1}-x_{2}-5 x_{3} & \geq 5 \\
5 x_{1}+5 x_{2}+x_{3} & \geq 25
\end{aligned}
$$

$$
x_{1}, x_{2}, x_{3} \geq 0
$$

(P6) Maximize: $Z=-3 x_{1}-4 x_{2}-2 x_{3}$

Subject to: $\quad x_{1}+x_{2} \leq 10$

$$
\begin{array}{r}
x_{1}+3 x_{2}+x_{3} \geq 9 \\
x_{2}+x_{3} \geq 4 \\
x_{1}, x_{2}, x_{3} \geq 0
\end{array}
$$

\section{Conclusion}

In this paper, the algorithm developed has been tested on some problems as stated in section 4.0 of this paper and the corresponding results are shown in tables below. From the tables, while some results are very close to the analytical results, some are equal to the analytical results which invariably justify clearly the applicability of the matrix-vector operations techniques in solving constrained optimization problems. Note: $s_{i}$ 's $(i=1, \ldots, n)$ are slack/surplus variables.

\section{References}

[1]. RAO, S. S., (1978), Optimization Theory and Applications, Willy and Sons.

[2]. THOMAS, F.E., and DAVID, M.H.,(2001), Optimization of Chemical Processes, McGraw Hill

[3]. OKONTA, P. N. and J. S. APANAPUDOR,(2001), Undergraduate Linear Algebra, Functional Publishing Company, Surulere, Lagos State. Nigeria.

[4]. RICHARD BRONSON and GOVINDASAMA NAADIMUTHU.(2007), Operations Research, Tata McGraw-Hill Publishing Company Limited, New Delhi.

[5]. SEYMOUR LIPSCHUTZ and MARC LIPSON,(2009), Linear Algebra, McGraw-Hill Companies, New Delhi.

[6]. GUPTA P. K. and HIRA D. S. (2012), Operations Research, S. Chand \& Company Ltd, New Delhi.

Table of Results for $(\mathrm{P} 1)$

\begin{tabular}{|l|l|l|l|l|l|l|}
\hline Iterations & $x_{1}$ & \multicolumn{1}{c|}{$x_{2}$} & \multicolumn{1}{c|}{$x_{3}$} & \multicolumn{1}{c|}{$s_{1}$} & \multicolumn{1}{c|}{$s_{2}$} & $s_{3}$ \\
\hline 1 & 0 & 0 & 0 & 240 & 156.6667 & 430 \\
\hline 2 & 0 & 0 & 156.6666667 & 126.66666667 & 0 & 430 \\
\hline 3 & 0 & 42.22222222 & 156.6666667 & 0 & 0 & 218.8888889 \\
\hline
\end{tabular}

Analytical Result: $x_{1}=0, x_{2}=42.22222222, x_{3}=156.6666667$

Table of Results for (P2)

\begin{tabular}{|l|l|l|l|l|l|l|l|}
\hline Iterations & \multicolumn{1}{|c|}{$x_{1}$} & \multicolumn{1}{|c|}{$x_{2}$} & \multicolumn{1}{c|}{$x_{3}$} & $x_{4}$ & $s_{1}$ & $S_{2}$ & $s_{3}$ \\
\hline 1 & 0 & 0 & 0 & 0 & 6 & 8 & 4 \\
\hline 2 & 0 & 0.6666667 & 0 & 0 & 4 & 6 & 0 \\
\hline 3 & 0 & 0.5454545455 & 0.72727277273 & 0 & 0 & 2.727272727 & 0 \\
\hline
\end{tabular}

Analytical Result: $x_{1}=0, x_{2}=2.666666667, x_{3}=0, x_{4}=0$

Table of Results for(P3)

\begin{tabular}{|l|l|l|l|l|l|l|l|}
\hline Iterations & \multicolumn{1}{|c|}{$x_{1}$} & \multicolumn{1}{|c|}{$x_{2}$} & \multicolumn{1}{|c|}{$x_{3}$} & \multicolumn{1}{c|}{$s_{1}$} & \multicolumn{1}{c|}{$s_{2}$} & \multicolumn{1}{c|}{$S_{3}$} & \multicolumn{1}{c|}{$s_{4}$} \\
\hline 1 & 0 & 0 & 0 & 10 & -10 & 15 & -15 \\
\hline 2 & 0 & -5 & 0 & -20 & 20 & 0 & 0 \\
\hline 3 & 0 & 1.666666667 & -20 & -20 & 0 & 0 & 0 \\
\hline
\end{tabular}

Analytical Result: $x_{1}=6.666666667, x_{2}=0.555555556, x_{3}=0$ 
Table of Results for (P4)

\begin{tabular}{|l|l|l|l|l|l|l|l|}
\hline Iterations & \multicolumn{1}{|c|}{$x_{1}$} & \multicolumn{1}{|c|}{$x_{2}$} & \multicolumn{1}{c|}{$x_{3}$} & \multicolumn{1}{c|}{$x_{4}$} & \multicolumn{1}{c|}{$s_{1}$} & $S_{2}$ & \multicolumn{1}{c|}{$S_{3}$} \\
\hline 1 & 0 & 0 & 0 & 0 & 10 & 5 & 6 \\
\hline 2 & 0 & 0 & 0 & 2.5 & 7.5 & 0 & 4.75 \\
\hline 3 & 1.071428571 & 0 & 0 & 0.7142857143 & 0 & 0 & -4.214285714 \\
\hline
\end{tabular}

Analytical Result: $x_{1}=1.071428571, x_{2}=x_{3}=0, x_{4}=0.714285714$

Table of Results for (P5)

\begin{tabular}{|l|l|l|l|l|l|l|}
\hline Iterations & \multicolumn{1}{|c|}{$x_{1}$} & \multicolumn{1}{|c|}{$x_{2}$} & \multicolumn{1}{c|}{$x_{3}$} & $s_{1}$ & $s_{2}$ & $s_{3}$ \\
\hline 1 & 0 & 0 & 0 & 10 & 9 & 4 \\
\hline 2 & 0 & 3 & 0 & -21 & 0 & 1 \\
\hline 3 & 0 & 2.5 & 1.5 & 7.5 & 0 & 0 \\
\hline
\end{tabular}

Analytical Result: $x_{1}=7.5, x_{2}=0, x_{3}=0.5$

Table of Results for (P6)

\begin{tabular}{|l|l|l|l|l|l|l|}
\hline Iterations & \multicolumn{1}{|c|}{$x_{1}$} & \multicolumn{1}{|c|}{$x_{2}$} & \multicolumn{1}{|c|}{$x_{3}$} & \multicolumn{1}{|c|}{$s_{1}$} & \multicolumn{1}{c|}{$s_{2}$} & \multicolumn{1}{c|}{$S_{3}$} \\
\hline 1 & 0 & 0 & 0 & -8 & -5 & -25 \\
\hline 2 & 0 & 5 & 0 & -3 & -10 & 0 \\
\hline 3 & 0 & 4.25 & 3.75 & 0 & -28 & 0 \\
\hline
\end{tabular}

Analytical Result: $x_{1}=0, x_{2}=4, x_{3}=0$ 